\title{
INTELLIGENT COMPUTING FOR THE MANAGEMENT OF CHANGES IN INDUSTRIAL ENGINEERING MODELING PROCESSES
}

\author{
László HoRváth, Imre J. Rudas, János F. Bitó \\ John von Neumann Faculty of Informatics \\ Budapest Tech Polytechnical Institution \\ Népszínház u. 8, H-1081 \\ Budapest, Hungary \\ e-mail: laszlo.horvath@nik.bmf.hu, rudas@bmf.hu, bito.janos@nik.bmf.hu
}

\section{Gerhard HANCKE}

Department of Electrical, Electronic and Computer Engineering

University of Pretoria

Lynnwood Road, Pretoria 0002

Pretoria, South Africa

e-mail: gerhard.hancke@eng.up.ac.za

Revised manuscript received 12 October 2005

\begin{abstract}
Advancements in engineering modeling have changed the work of engineers during the last two decades. Sophisticated descriptions store information about shape oriented engineering objects and their relationships. Boundary representations of form features constitute shape models. Rules and checks have replaced simple data form of shape model entity attributes. This change of modeling facilitates a next step towards application of computer intelligence at engineering object related decisions. The authors propose a method of intelligent attribute definition for integrated decision assistance environments of modeling systems. This method provides quick and comprehensive assessment of situations for decisions on modification of modeled objects in very complex information environments. The paper starts with an outline of the approach to intelligent decision assistance by the authors. Next, an Internet portal communicated scenario of the proposed modeling
\end{abstract}


is discussed. Following this, multilevel solution for modeling, adding characteristics for engineering objects, and definitions and communications are detailed as essential methods in the proposed modeling. Finally, behaviors for essential classes of modeled objects and an example for the definition of situations and behaviors represent implementation issues.

Keywords: Industrial modeling

\section{INTRODUCTION}

Engineering for products has been extended into a continuous activity in comprehensive product modeling systems. Most products allow application of shape descriptions with other information linked to shape information. Advanced modeling describes interrelated product data for easy modification of modeled information when change of an engineering object generates the need for modification of a set of related objects. Appropriateness of a change decided for an engineering object must be evaluated for all of the related objects.

It is very difficult to assess the consequences of a design change. Less or more decisions must be changed with many additional consequences to be assessed. Conventional analysis of data connections is not enough. In recent CAD/CAM systems, engineers can define and then use knowledge in formulas, rules, and checks. Rule and check bases are established and available to ensure design consistency as well as compliance with company and partner specifications, standards, practices, etc. They are useful tools to avoid high number of design iterations and redesign. In most advanced industrial modeling systems, optimizing is available for best appropriate combinations of parameters of modeled objects [1].

In this paper, the authors propose a method for integration of intelligence in engineering modeling as a contribution to enhancement of present modeling in CAD/CAM systems. The proposed method fits into the above discussed features of recent industrial modeling. Preliminary projects resulted in approaches and methodologies for the reported work. The authors analyzed information structure of engineering models [2]. They analyzed possible roles of behavior definitions and adaptive functions in decisions of engineers [3]. All of the interrelated decisions in engineering are of human origin. This is why modeling of human intent as background of engineering decisions [4], and integration of human intent modeling in engineering modeling [5] were the main issues in the modeling by the authors.

Activities of the authors in modeling, handling and propagation of changes of engineering objects [6] and in application of environment adaptivity in engineering modeling [7] are recent preliminaries of the proposed method.

The paper starts with an outline of the approach to intelligent decision assistance by the authors. Next, an Internet portal communicated scenario of the proposed modeling is discussed. Following this, multilevel solution for modeling, adding cha- 
racteristics for engineering objects, and definitions and communications are detailed as essential methods in the proposed modeling. Finally, behaviors for essential classes of modeled objects and an example for the definition of situations and behaviors represent the implementation issues.

\section{ENGINEERING PROCESS BASED INTELLIGENCE}

Any information about modeled objects in a product model is recorded as a modification that affects other information. Essential content of a model can be divided into three groups (Figure 1). There are data for the description of engineering objects by using appropriate representation. Knowledgeware includes knowledge for modeling and modeled objects. Associativities connect model data and knowledge information. Development, revision or upgrade of any of these groups is considered as modification. Modification may be initialized by a human or by a procedure. Human applies intent while procedure applies situation based behavior analysis. Modification is executed by adaptivity. Adaptivity is an entity to describe change of one or more modeled entities. For example, diameter, length, heat treatment or surface finish of a shaft in a mechanical drive unit has been changed. This change requires additional modifications because it affects assembly, other parts, behavior of the drive, manufacturing process, costs of material, and manufacturing, etc. Consequently, modification carried by adaptivity can be considered as only an attempt for modification. Adaptivity is executed when it is accepted.

High proportion of engineering objects in the practice can be handled by shapecentered modeling where non-shape information is attached to shape information. The authors restricted the area of their analyses into shape centered product modeling. Product model serves development, production planning, manufacturing planning, manufacturing, application, and recycling of a product.

Recent trend in product modeling can be described by several focus methods and techniques. The proposed modeling assumes them. Product information is carried by elementary, structural and relationship model entities. Reference models, resources, and application protocols are essential elements of integrated product modeling [8]. Behavior description [9] and agent [10] in models represent an initial stage of intelligent engineering modeling. Description of form features [11] and surfaces [12] in boundary representation represents an outstanding development of shape modeling in the last decade.

Integration of the proposed modeling into advanced engineering systems is shown in Figure 2. Group work of engineers is organized around a portal with wide range of Internet and collaborative functions. Engineers E1i - Eni are participants of a project based work group in a product related engineering environment and perform engineering processes. Engineers access resources through Internet portal functions by using specially configured browsers. An engineering process comprises large number of coordinated proposals, counterproposals, decisions, and modifications about model entities and their parameters in order to develop, modify, correct and 


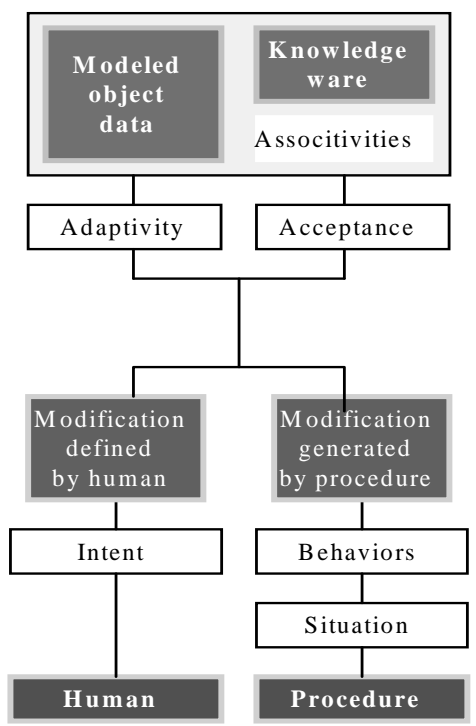

Fig. 1. Intelligent decision assistance

variant definition of products. Conventional modeling uses procedures, model descriptions, and product data management (PDM). Conventional product modeling processes, product data management and product data are accessed as conventional computer aided engineering resources. Decision based change management is in the center of intelligent engineering processes. In the approach and method proposed by the authors, intelligent engineering processes are supported by descriptions of behaviors of modeled objects, adaptivities and intent of engineers. Human intent originated knowledge is applied to analyze behavior and to assist decision processes by adaptive actions.

\section{METHODS IN THE PROPOSED MODELING}

The present product modeling relies upon interrelated computer representations. Shape oriented models are composed by shape modification form features. Shape is represented by topology-defined structure of curves and surfaces. The authors proposed an extension of feature entities into the area of intelligent content of model. Behavior and adaptivity features have been defined and processed in a four-leveled modeling (Figure 3). On level one, behavior features are created for actual situations. Following this, inside and outside adaptivity features are generated under the control of behavior features. Adaptivity features carry information along associativity links about attempts for modification of model objects. On level two, inside adaptivity features are generated and applied for modification of entities inside of the actual model object as a consequence of changes communicated with 


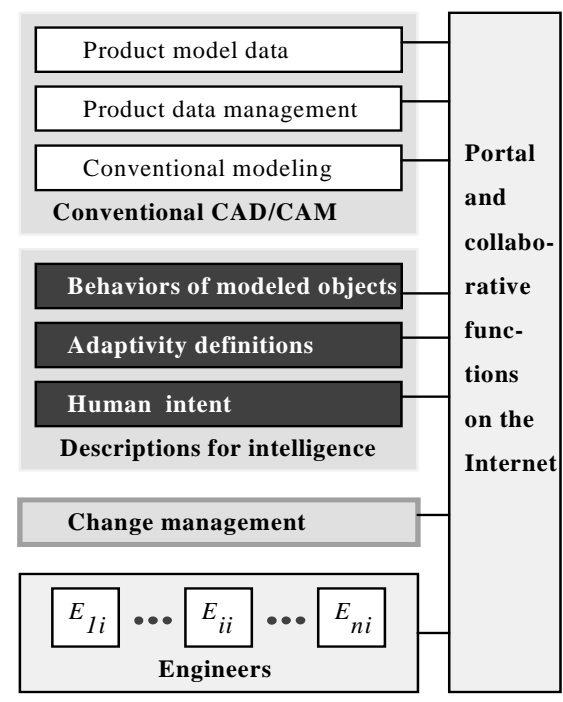

Fig. 2. Scenario of the proposed modeling

them. On level three outside adaptivity features are generated and applied for making attempts to modify model entities outside of the integrated model object. Sometimes, a behavior feature creates demand for modifications of non-associative entities. If it is possible, new associativity definitions are defined then the processing steps back to level three. Outside adaptivity features utilize new associativity definitions. Unaccepted adaptivities are undergone to conflict handling process. Unsuccessful attempt in definition of associativity means the entities inaccessible for the intelligent system. One of the conventional ways of sending change messages for engineers who are responsible for the non-cooperative models is to be applied. Human response to these messages is required to substitute adaptive automatism by conventional human activity.

Characteristics of a modeled object are established as a combination of parameters. The best combination depends on design objectives and interactions between parameters within and outside of the object. Tuning of too many parameters is difficult so that the main influential parameters must be identified within the affected zone of a modeled object parameter. Main object characteristic related issues are establishing an initial structure of model object, identification of parameters affected by a design change, and predicting significant influences in the affected zone. Moreover, sometimes a need for extending of an actual affected zone complicates the modeling.

The authors propose initial characteristics of an actual simple or complex modeled object to be created by instancing generic object definitions (Figure 4). Other object characteristics are added to objects during their lifecycle by self-adaptivity management and inheritance. Adaptivity processes within a model object are con- 


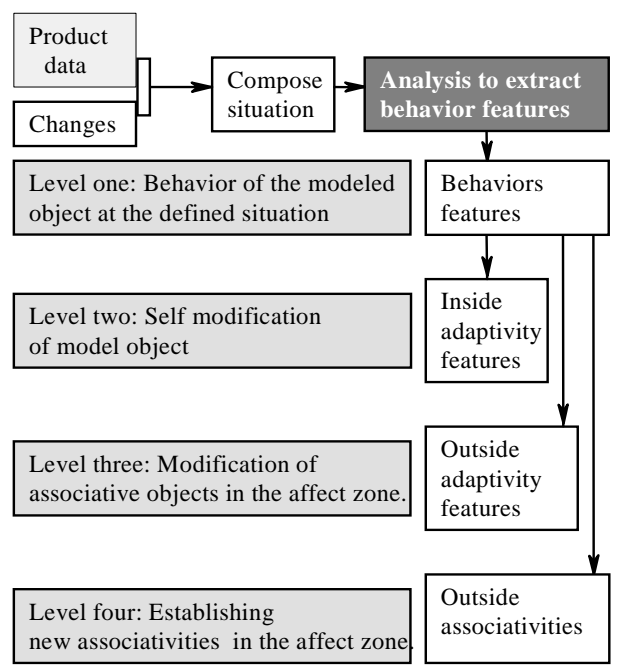

Fig. 3. A multilevel solution for modeling

trolled by behavior based analysis. Creation and handling of elementary object, structure, and relationship representations are controlled by actual object characteristics. Definition and application of behaviors are supported by purposeful knowledge representation. The world outside of an actual model object is interfaced by outside links. Outside effects are received and processed by behavior analysis. Specific environment adaptivity is established by effect on other object management. Any communicated change of the related outside world objects initiates behavior based evaluation. An evaluation creates new changes to initiate new evaluations. Changes of related objects, specification, knowledge, experience and human intervention are processed by evaluation. Humans have responsibility-based privileges.

Conventional model data and intelligent content are integrated in model objects. In the center of an integrated model object are the managers for control of engineering processes (Figure 5). Roles and types of managers are discussed by the authors in [6]. A passport element of an integrated model object describes status, acceptance, permission, and other access and application related information. Main elements of an integrated model object are definitions, instances, tracking changes, and communications. Inside and outside communication are organized along chains of associativities. In other words, an integrated model object uses associativity definitions for control and processing of inside and outside communication. Chains of associativities are developed step by step during the lifecycle of an integrated model object according to demands of behavior driven adaptivity actions.

New entity definitions, new instances of earlier defined entities, and new values of entity attributes are tracked across associative entities. Associativities constitute bridges between entities and attributes to propagate effects of changes. There are 


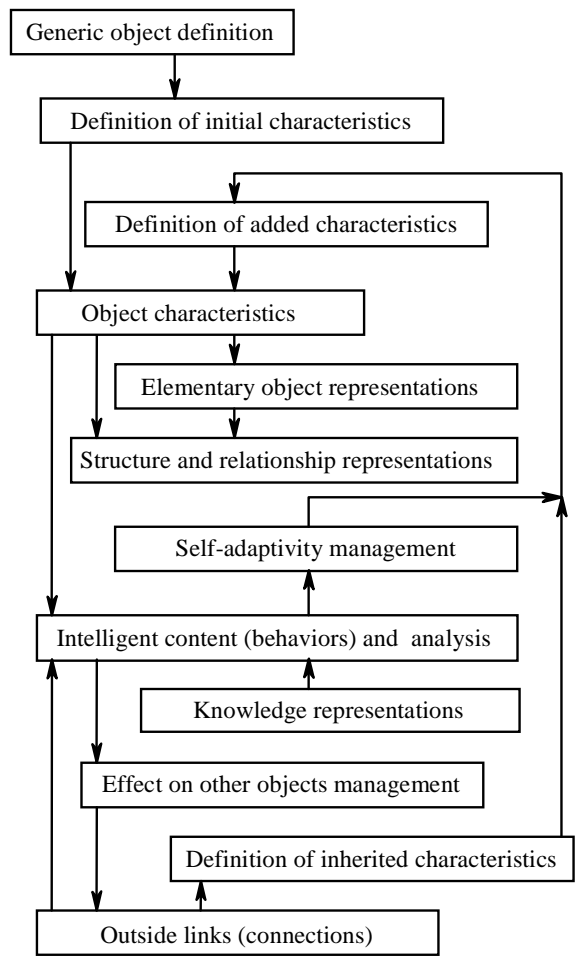

Fig. 4. Adding characteristics for engineering objects

defined, known but to be defined, and unknown associativities. Associative outside world comprises both cooperative and non-cooperative models. Cooperative models are able to communicate mutual adaptive processes. Non-cooperative models are conventional ones that can accept authorized modifications and make access of model data available for the cooperative environment. As an auxiliary function, communication also can be done by conventional data exchange with non- associative modeling systems.

Any change is processed according to its effect on other definitions, entities, and attributes. Entities describe engineering objects, knowledge, circumstances, and procedures. A behavior-based analysis of a change produces one of the nonaffected, affected, or affect unknown states of inside and outside associative entities and attributes.

Description of engineering objects and their behaviors rely upon definitions. Figure 5 relates main categories of definitions. Engineering objects are described by entities. A solution comprises a set of entities representing interrelated engineering objects for a well-defined engineering purpose. Engineering object is associated with behavior definitions according to the design goals associated with them. 


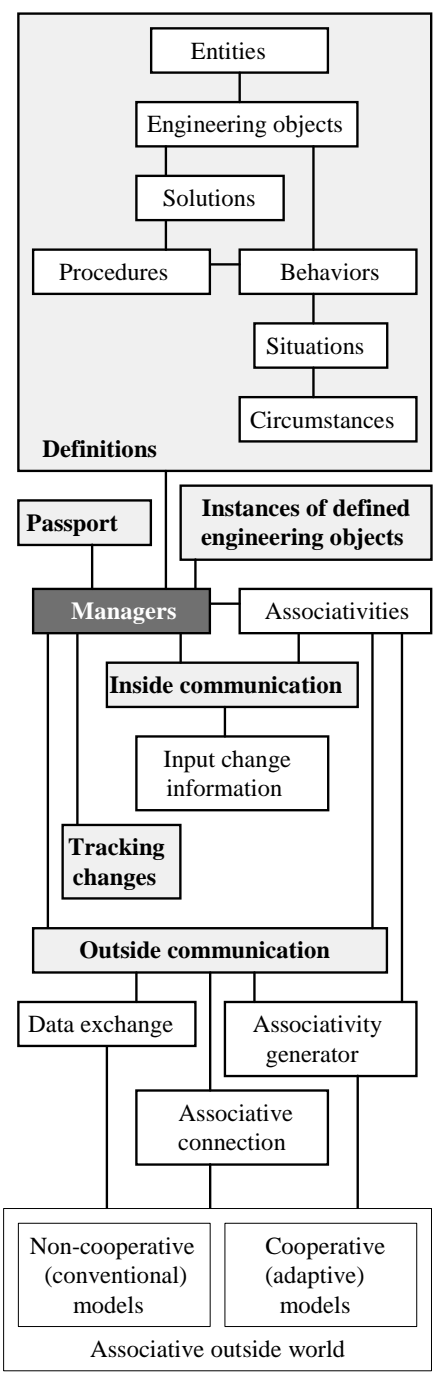

Fig. 5. Definitions and communications

Behaviors are analyzed for situations. A situation is defied as a combination of circumstances.

Mechanism of environment adaptivity of an integrated model object ensures change information to wake up all related associativities. Some modifications are permitted without any analysis while others need previous analysis. Relevant definitions, instances, circumstances, situations, and behaviors are retrieved during preprocessing for analysis. 


\section{IMPLEMENTATION IN INDUSTRIAL MODELING}

In present industrial practice, well-established and well-proven structure is applied for shape-centered models. Including intelligent content in these models is a primary trend in recent CAD/CAM. Model of a mechanical system is outlined in Figure 6 by conventional and feature extension entities and their connections.

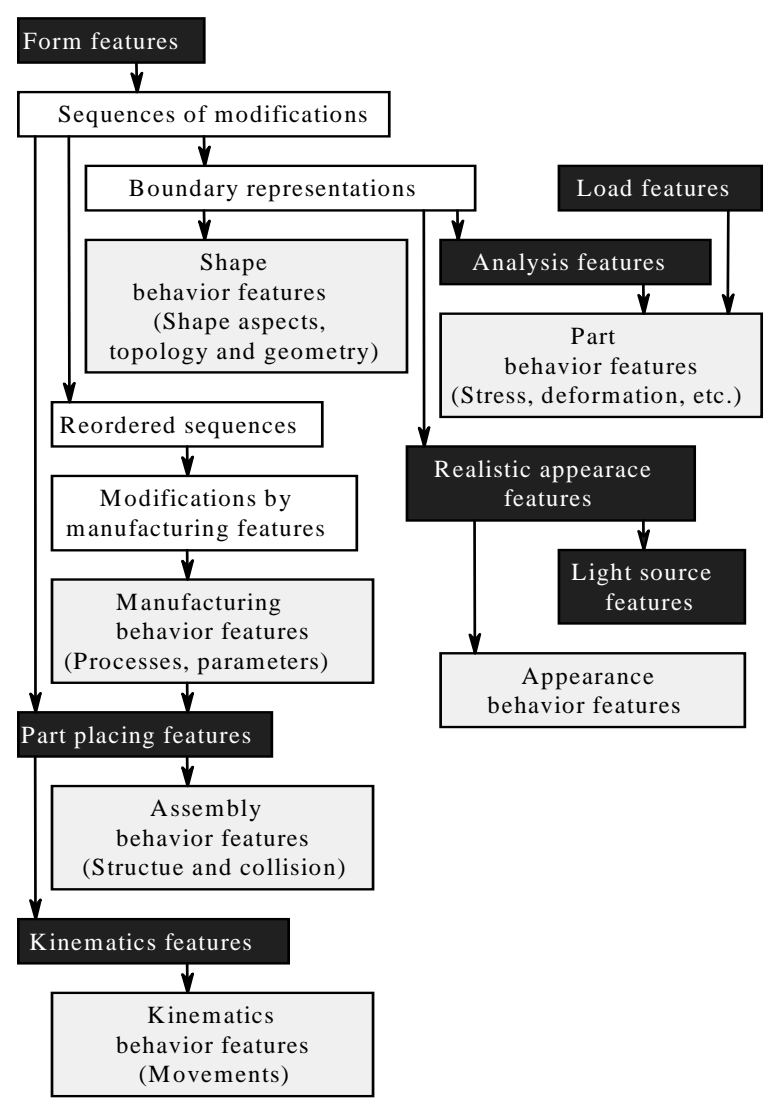

Fig. 6. Behaviors for essential classes of modeled objects

Associativities are defined along these connections. Elementary and structure features and their connections describe modeled objects while behavior features describe behaviors of modeled objects. Groups of features in dark boxes are elementary ones, and are selected, modified and defined by using generic and instance feature libraries. The white boxes show structure and relationship features. Behavior features in the gray boxes are attached to object description features. Essential behavior features are attached to shape, part, manufacturing, assembly, kinematics, and appearance features. 
Behavior specifications represent design objectives and originate from customer demands, requirements by engineering activities, experiences, and personal intents. Behavior feature answers behaviors of the related modeled object at a predefined circumstance. Active behaviors define parameters of the modeled objects while passive ones serve for comparison of specified and actual behaviors. Figure 7 shows an example for implementation of the proposed behavior based assistance of decisions. A self locking conical connection is created by design of two parts, Part A and Part B. Size of the cone depends on version of Part A. On the other hand, version of Part A depends on version of Part B. Four situations are defined for four behaviors. All behaviors must be effective to serve parallel design goals. Structure behavior depends on actual parts while connection behavior depends on situation of placing of mated surfaces. Collision avoidance behavior is analyzed for moving volumes of parts. Finally, self-locking behavior requires appropriate cones.

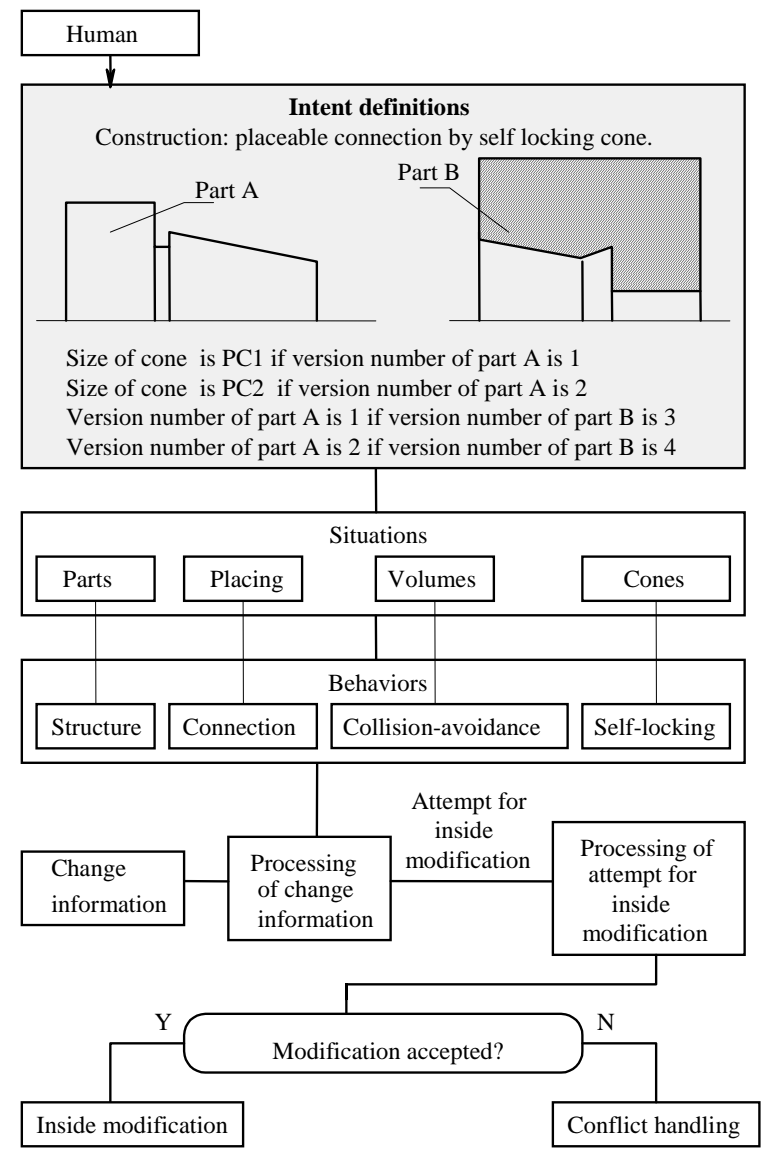

Fig. 7. Example for situations and behaviors 
Change information initializes behavior-based analysis and may generate an attempt for inside modification. Repeated analysis of one or more behaviors of some modeled objects is necessary in these cases. As an example, one of the parts must be replaced by a new part. The newly included part causes collision and cannot provide self-locking. However, the related two modifications of the new part are attempted unsuccessfully. Because attempts for necessary modification of the new part are refused, new analysis and resolution of the conflict are necessary.

\section{SUMMARY}

The paper introduces a method for integration of intelligence in engineering modeling as a contribution to enhancement of present modeling for CAD/CAM systems in shape centered product design. The proposed method supports assess of consequences of design changes where less or more decisions are changed and many additional consequences may be encountered. Recent knowledge based modeling for CAD/CAM systems do not offer efficient solution for this problem. Multilevel modeling, adding characteristics for engineering objects, and specific definitions and communications are the essential methods in the proposed modeling. Feature entity definitions have been extended to the area of intelligent content of model. Initial characteristics of an actual simple or complex modeled object are created by instancing of generic object definitions. Quick and comprehensive assessment of situation at decisions on modification of modeled objects is assisted by analysis of behaviors for situations composed by circumstances. Behavior and adaptivity features are defined. They are processed in a four leveled modeling in an integrated solution with conventional descriptive modeling.

\section{Acknowledgment}

The authors would like thank Hungarian - South African Intergovernmental Science\& Technology Cooperation Programmes for the financial support (project No. DAK-3/02). The authors also gratefully acknowledge the grant provided by the OTKA Fund for Research of the Hungarian Government (project No. T 037304).

\section{REFERENCES}

[1] Xue, D.-Yang, H.: A Concurrent Engineering-Oriented Design Database Representation Model. Computer-Aided Design, Vol. 36, 2004, No. 10, pp. 947-965.

[2] Horváth, L.-Rudas, I. J.: Virtual Technology Based Associative Integration of Modeling of Mechanical Parts. Journal of Advanced Computational Intelligence, Vol. 5, 2001, No. 5, pp. 269-278.

[3] Horváth, L.-Rudas, I. J.-Hancke, G.: Feature Driven Integrated Product and Robot Assembly Modeling. In Proc. of The Seventh International Conference on Automation Technology, Automation 2003, Chia-yi, Taiwan, 2003, pp. 906-911. 
[4] Horváth, L.-Rudas, I. J.: Modeling of the Background of Human Activities in Engineering Modeling. Proceedings of the IECON '01, $27^{\text {th }}$ Annual Conference of the IEEE Industrial Electronics Society, Denver, Colorado, USA, pp. 273-278.

[5] Horváth, L.-Rudas, I. J.-Couto, C.: Integration of Human Intent Model Descriptions in Product Models. In Digital Enterprise - New Challenges Life-Cycle Approach in Management and Production, Kluwer Academic Publishers, pp. 1-12.

[6] Horváth, L.-Rudas, I. J.: Possibilities for Application of Associative Objects with Built-in Intelligence in Engineering Modeling. In Journal of Advanced Computational Intelligene and Intelligent Informatics, Tokyo, Vol. 8, 2004, No. 5, pp. 544-551.

[7] Horváth, L.—Rudas, I. J.-Hancke, G.—SzakÁL, A.: Integrated Environment-Adaptive Virtual Model Objects for Product Modeling. In Proc. of the 2003 IEEE International Conference on Systems, Man \& Cybernetics, Omnipress, Washington, D.C., USA., ISBN 0-7803-7953-5, IEEE Catalog Number 03CH37483C, pp. $498-503$.

[8] Mannistö, T.-Peltonen, H.-Martio, A.-Sulonen, R.: Modeling Generic Product Structures in STEP. Computer-Aided Design, Vol. 30, 1998, No. 14, pp. 1111-1118.

[9] Hasegawa, Y.-Fukuda, T.: Motion Coordination of Behavior-based Controller for Brachiation Robot. In Proceedings of the 1999 IEEE International Conference on Systems, Man, and Cybernetic, Human Communication and Cybernetics, IEEE, Tokyo, Vol. 6, 1999, pp. 896-901.

[10] Tambe, M.-Johnson, W. L.-Jones, R.-Koss, F.-Laird, J.Rosenbloom, P.-Schwamb, K.: Intelligent Agents for Interactive Simulation Environments. AI Magazine, Vol. 16, 1995, No. 1.

[11] Shah, J. J.-Mantyla, M.-Shah, J. J.: Parametric and Feature-Based CAD/CAM: Concepts, Techniques, and Applications. John Wiley \& Sons, 1995.

[12] Freitag, S.-Opitz, K.: Enhancements in Blending Algorithms. Hewlett-Packard Journal, October 1995, pp. 24-34.

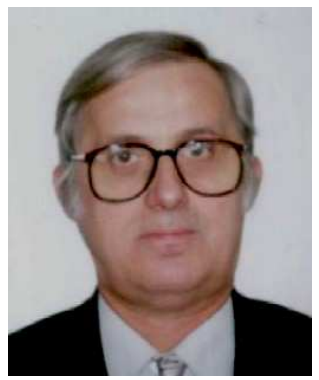

László HoRVÁTH received a Master Degree in Production Engineering from the Technical University of Budapest in 1971, the Dr. Techn. Degree in computer-aided process planning systems from the Technical University of Budapest in 1984 and the $\mathrm{Ph}$. D. degree in knowledge based process planning systems from the Hungarian Academy of Sciences in 1992. He is active as the Deputy Director for Sciences of the John von Neumann Faculty of informatics, Budapest Tech Polytechnical Institution and as a professor of Department of Mathematical and Computational Sciences. He currently teaches courses in CAD/CAM systems engineering, informatics and knowledge engineering. He is a Senior Member of IEEE. His present areas of research activity are: Computer Modeling of Design Intent, Environment-adaptive Intelligent Product Models, Methods for Computer Description of Engineering Objects, Integrated Robot and Engineering Systems and Virtual University. He has published more than 170 papers in various journals and international conference proceedings. 


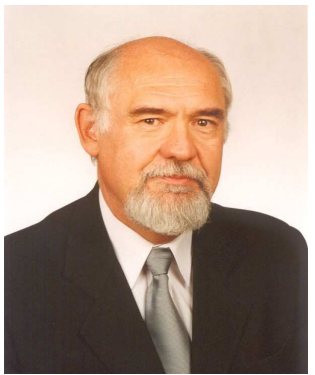

Imre J. RuDAS graduated from Bánki Donát Polytechnic, Budapest in 1971, received the Master Degree in Mathematics from the Eötvös Loránd University, Budapest, the Ph. D. in Robotics from the Hungarian Academy of Sciences in 1987, while the Doctor of Science degree from the Hungarian Academy of Sciences. He is active as a full university professor and Head of Department of Intelligent Engineering Systems. He serves as the Rector of Budapest Tech from August 1, 2003 for a period of four years. He is Vice President of IFSA for Membership Development. He is the President of the Hungarian Fuzzy Association and Steering Committee Member of the Hungarian Robotics Association and the John von Neumann Computer Society. He serves as an associate editor of IEEE Transactions on Industrial Electronics, member of editorial board of Journal of Advanced Computational Intelligence and Control Engineering Practice, member of various national and international scientific committees. He is the founder of the IEEE International Conference Series on Intelligent Engineering Systems and IEEE International Conference on Computational Cybernetics, and some regional symposia. He has served as General Chairman and Program Chairman of numerous scientific international conferences. His present areas of research activity are: Robotics with special emphasis on Robot Control, Soft Computing, Computed Aided Process Planning, Fuzzy Control and Fuzzy Sets. He has published one book, more than 300 papers in various journals and international conference proceedings.

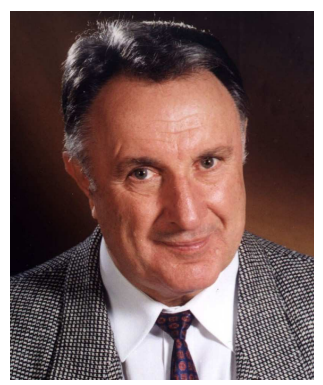

János F. Bıтó received the M. Sc. in mathematics and physics from József Attila University, Szeged (1958), Ph. D. at the Hungarian Academy of Sciences (1967), Doctor of Technical Sciences (D. Sc.), at the Hungarian Academy of Sciences (1971), Professor of physics at Eötvös Lóránd University (1986), at the Pázmány Péter Catholic University (2000), Budapest Tech (2002). Fellow grade Member of IEEE (1999) chairman of Committee for Information Technology of Hungarian Academy of Sciences, (2000-2006); member of the National Committee for Accreditation of Hungarian Universities and Colleges in the field of Electrical Engineering and Information Technology (1996-); member of the Committee for Habilitation at the Technical University of Budapest (1996-), at the Pázmány Péter Catholic University (1996), at Veszprém University (1997-), and at the Faculties of Electrical Engineering and Information Technology of the Technical University of Budapest(1996-); He has published about 225 scientific papers and 5 university guidebooks, over 200 papers and 5 books for science propagation in the fields as follows: electron and plasma physics, optoelectronic sensors, optical signal processing, physical foundations of robot control strategies, Artificial Neural Network (ANN) research for control and pattern recognition tasks, application of Information and Communication Technology. 


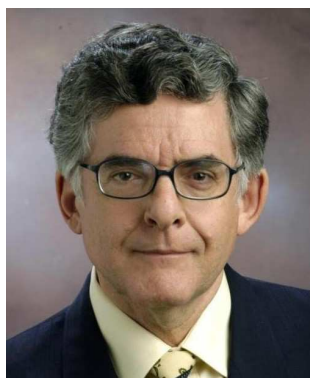

Gerhard HANCKE (SMIEEE) received the B.Sc., B. Eng. and M. Eng. from the University of Stellenbosch and the DEng (1983) from the University of Pretoria, where he is currently Chair of the Computer Engineering Program and the Research Group for Computer Networks and Security in the Department of Electrical, Electronic and Computer Engineering. His research interests are in wireless sensors and actuators networks and he partakes extensively in collaborative programs with research institutions internationally. He is a Professional Engineer and held offices in various international scientific and professional bodies. 